\title{
A dual flow bioreactor with controlled mechanical stimulation for cartilage tissue engineering
}

\author{
Authors \\ Spitters TWGM ${ }^{1}$, Leijten $\mathrm{JCH}^{1}$, Deus FD², Costa IBF${ }^{2}$, van Apeldoorn AA ${ }^{1}$, \\ Blitterswijk $\mathrm{CA}^{2}$, Karperien $\mathrm{M}^{1^{*}}$
}

\begin{abstract}
Author affiliation
${ }^{1}$ Dept. of Developmental BioEngineering, MIRA Institute for Biomedical Technology and Technical Medicine, University of Twente, The Netherlands

${ }^{2}$ Dept. of Tissue Regeneration, MIRA Institute for Biomedical Technology and Technical Medicine, University of Twente, The Netherlands
\end{abstract}

\section{Author Contribution}

Study design; TS, JL, AA and MK. Experimentation and data interpretation TS, JL, FD, IC. TS wrote the manuscript with help of all authors. MK supervised the study.

${ }^{*}$ Correspondence to: M. Karperien, Dept. of Developmental BioEngineering, MIRA Institute for Biomedical Technology and Technical Medicine, University of Twente, Drienerlolaan 5, 7500 AE Enschede, PO box 217, The Netherlands. E-mail: h.b.j.karperien@utwente.nl 


\begin{abstract}
In cartilage tissue engineering bioreactors can create a controlled environment to study chondrocyte behavior under mechanical stimulation or produce chondrogenic grafts of clinically relevant size. Here we present a novel bioreactor, which combines mechanical stimulation with a two compartment system through which nutrients can be supplied solely by diffusion from opposite sides of a tissue engineered construct. This design is based on the hypothesis that creating gradients of nutrients, growth factors and growth factor antagonists can aid in the generation of zonal tissue engineered cartilage. Computational modeling predicted that the design facilitates the creation of a biologically relevant glucose gradient. This was confirmed by quantitative glucose measurements in cartilage explants. In this system it is not only possible to create gradients of nutrients, but also of anabolic or catabolic factors. Therefore, the bioreactor design allows control over nutrient supply and mechanical stimulation useful for in vitro generation of cartilage constructs that can be used for the resurfacing of articulated joints or as a model for studying OA disease progression.
\end{abstract}

Keywords: Dual compartment, bioreactor, gradients, mechanical stimulation, cartilage tissue engineering 


\section{Introduction}

If left untreated, critical size cartilage defects will lead to degenerative diseases like osteoarthritis (OA) [1, 2]. Clinical techniques such as autologous chondrocyte implantation $(\mathrm{ACl})$, matrix-induced chondrocyte implantation and microfracture have demonstrated to improve healing of critical size defects [3-5]. Although the mentioned techniques have shown positive results, the regenerated cartilaginous tissue is of less quality and neo-tissue integration with existing cartilage remains challenging [611]. An alternative strategy is the generation of engineered tissues of clinically relevant amount [12] or size [13] in vitro, which can be used for surface defect repair. For the production of such tissue engineered constructs, bioreactor systems are essential, as they can provide control over the in vitro environment and allow for creating optimal conditions for neo-tissue formation [14].

Cartilage can be considered as a simple tissue, as it only contains one cell type and only two major extracellular matrix (ECM) components, proteoglycans and collagen type II. However, it is complex in the sense that it is a layered anisotropic structure which is required to absorb strong mechanical forces, distribute load and lubricate the joint $[15,16]$. These functions arise in part from the zonal organization of the tissue. Collagen fibers, responsible for absorbing and distributing the load, are orientated parallel along the synovial surface and become perpendicularly orientated with depth anchoring in the subchondral bone plate. This results in a fountain-like structure with the purpose of distributing load and retrieving the tissue's original shape [17]. Glycosaminoglycans (GAGs) are distributed inhomogeneously as the articular surface is almost void of these molecules and their concentration increases with depth [18]. Water is attracted by the negatively charged proteoglycans. Upon deformation, water is expelled from the tissue, which flows back when the tissue 
relaxes. Although several bioreactor studies have demonstrated the ability to stimulate sulphated glycosamoniglycan (sGAG) deposition, a native distribution of sGAGs in tissue engineered constructs has yet to be obtained. In addition, the fountain-like structure of collagen fibers has so far not been generated in vitro. As a result it remains a challenge to engineer cartilaginous tissue in vitro that possesses the mechanical properties of native articular cartilage.

Several bioreactors for cartilage tissue engineering have been described. In these system nutrients are usually supplied through perfusion, creating shear stress, or static-like culture as in a culture dish. However, articular cartilage is not subjected to an external active flow and except for the perichondrium it does not receive nutrients from the sides. It is situated between synovial fluid and the subchondral bone plate, which physically separates the articular cartilage from the bone marrow. It has been postulated that cartilage is supplied with nutrients from the subchondral bone and from the synovial fluid $[19,20]$. Applying this feature to in vitro culture was described by Chang et al, and can be mimicked in transwell cultures. However, no mechanical stimulation can be applied in this system [21]. Chondrocytes in their natural environment are subjected to mechanical load. Therefore, bioreactors for cartilage tissue engineering that are equipped with a compression module more closely resemble cartilage's in vivo environment. Load can either be applied by confined [22], sliding [23] or rotating compression [24]. Demarteau et al showed that under perfusion and confined compression sulphated glycosaminoglycan metabolism was increased in cell-loaded PEOT/PBT block co-polymer foam scaffolds, but this design did not originate in an inhomogenous SGAG distribution as in native cartilage [22]. Kock et al showed production of collagen II next to a homogenous distribution of sGAGs under sliding indentation, but this did not result in fountain like orientation of 
the collagen network [23]. The stimulatory effect of compression on the expression of zone specific genes was shown by Grad et al indicating the importance of mechanical stimulation in the regeneration of functional chondrogenic constructs in vitro $[25,26]$.

Here, we describe a bioreactor design in which nutrient supply from both the synovial and subchondral side can be mimicked. This configuration will facilitate formation of gradients of nutrients, growth factors and growth factor antagonists through the cartilage tissue. In addition, the bioreactor is complemented with options for confined compression. We hypothesize that the ability to create gradients of relevant factors combined with confined load will aid in the generation of neo-cartilage better resembling cartilage's natural organization and mechanical properties. In this study we introduce the basic design of a dual compartment, compression equipped novel bioreactor that can be used to engineer cartilage or osteochondral constructs in vitro. 


\section{Materials and methods}

\section{Bioreactor design}

The dynamic (Figure 1A) and static (Figure 1B) bioreactors were designed in Solidworks 2009, Edition SP4.1. The stainless steel housing of the dynamic bioreactor was produced by electrical discharge machining (EDM) effectively creating a construct chamber of $1 \mathrm{~cm}^{3}$ (Figure 1A). Removable round polymethylmethacrylaat (PMMA) windows were installed at the front and back side of the housing and allowed for placement of the construct into the chamber. Windows contained a nitrile butadiene rubber (NBR) O-ring ensuring a tight seal preventing leakage and infection.

The complete chamber has a height of $13 \mathrm{~mm}$ and a width and depth of $10 \mathrm{~mm}$. Explants were placed in a $4 \mathrm{~mm}$ high insert. Articular cartilage constructs were placed in a square insert (Figure 1C). Osteochondral plugs were placed in a round insert (Figure 1C). The inserts were placed in the reactor chamber on top of a $4,5 \mathrm{~mm}$ high perforated polycarbonate plate (Figure 1C, bottom) that allowed for diffusion of nutrients and metabolites. The square inserts were combined with a $4,5 \mathrm{~mm}$ high perforated metal compression insert and the round inserts were combined with a $7 \mathrm{~mm}$ diameter rounded Teflon head (Figure 1D). Above and below the inserts are medium reservoirs with an approximate volume of $270 \mu \mathrm{L}$.

Culture medium within the channels of the perforated plates is refreshed by diffusion from the top and bottom medium reservoirs. The medium reservoirs are in turn refreshed by laminar flow of medium from four $10 \mathrm{~mL}$ syringes (Hamilton, Reno, USA) which are driven by a syringe pump (kdScientific, Holliston, USA).

A stainless steel plunger is attached to the compression insert. This plunger applies top down compressional force on the tissue engineered constructs by means of a 
separate steppenmotor. The exerted force is continuously monitored by a Futek pressure sensor (model LSB200, type FSH00105) and is configured and controlled via labVIEW software (National Instruments, Austin, USA).

Additionally, we also designed a polycarbonate static bioreactor, which is identical to the dynamic bioreactor in the sense that samples of the same dimensions can be inserted and the volume of both medium reservoirs are identical to the dynamic bioreactor (Figure 1B). The inlets were provided with a screw rod to enable the insertion of this bioreactor in a dynamic system. The device was placed in a custommade four well plate during culture. The static bioreactor lacked modalities for mechanical compression.

\section{Material source}

Knees of 10-12 month old calves were collected from the local abattoir. Femoral condyles were sterily exposed by removing the muscles and patella.

For the articular cartilage explant model, cartilage slices were made with a custom made slicer (figure 1E, bottom) to obtain slices of equal height. Since this slicer could not slice through the calcified zone of the cartilage, it was ensured that slices did not contain bone contamination. Cubes of $4.5 \mathrm{~mm}$ by $4.5 \mathrm{~mm}$ by $1-1.4 \mathrm{~mm}$ in height were punched from the slices with a 4-frame stainless steel knife (figure 1C, top) and a puncher. The cubes were then transferred to the polycarbonate insert (Figure 1C, second from the top) and loaded into the bioreactor chamber together with the metal compression and perforated bottom inserts. Explants were always cultured with the synovial side oriented upwards and the subchondral side downwards. The 4 explants were consistent in size. The thickness of the explants depended on the region of harvesting (figure 1F). 
For the osteochondral model, $7 \mathrm{~mm}$ diameter osteochondral plugs were punched from the condyles. These explants were about $6 \mathrm{~mm}$ in height to assure that cartilage and bone were properly attached after explantation. Afterwards bone was removed to adjust the explant's height to $3 \mathrm{~mm}$ and they were transferred to a polycarbonate insert (Figure 1C, third from the top) and loaded into the chamber together with the Teflon compression and perforated bottom insert.

\section{Computational modeling}

Computational fluid dynamics (CFD) of the fluid flow and diffusion in culture conditions were set-up and solved in the MEMS module (microfluidics - flow with species transport - Incompressible-Navier Stokes) in Comsol Multiphysics version 3.5a software (Comsol, Zoetermeer, The Netherlands).

The Navier-Stokes equation that was solved for incompressible fluid dynamics is as defined by Eq. 1:

$$
\begin{aligned}
& \rho \frac{\partial u}{\partial t}=-\eta \nabla^{2} u+\rho(u \cdot \nabla) u+\nabla p=F \\
& \nabla \cdot u=0
\end{aligned}
$$

where $\rho$ is the fluid density $\left(\mathrm{kg} \mathrm{m}^{-3}\right), u$ is the velocity field $\left(\mathrm{m} \mathrm{s}^{-1}\right), t$ is the time $(\mathrm{s}), \eta$ is the dynamic viscosity of the fluid $\left(\mathrm{kg} \mathrm{m}^{-1} \mathrm{~s}^{-1}\right), \nabla$ is the del operator, $p$ is the pressure (Pa) and $F$ represents other forces (gravity or centrifugal force), which in this case equals 0 . It was assumed that medium could not flow through the cartilage explants. Fluid flow was set at a velocity of $2.65 \times 10^{-3} \mathrm{~m} / \mathrm{s}(=0.5 \mathrm{~mL} / \mathrm{min})$. Species transport was coupled to Navier-Stokes. Glucose and oxygen concentrations in the bioreactor chamber were modeled in steady state using the following assumptions i) Walls in different conditions were considered rigid and impermeable, ii) no-slip boundary conditions were applied to surfaces, iii) the glucose diffusion 
constant $(\theta)$ in water was set to $9.0 \times 10^{-10} \mathrm{~m}^{2} / \mathrm{s}$ and in cartilage was $3.0 \times 10^{-10} \mathrm{~m}^{2} / \mathrm{s}$ $[27,28]$. Initial concentration of glucose in the top compartment was $25 \mathrm{mmol} / \mathrm{L}$, representing high glucose DMEM, and was $0 \mathrm{mmol} / \mathrm{L}$ in the bottom compartment, representing DMEM without glucose. The glucose consumption rate of chondrocytes in cartilage was considered to be $0.62 \times 10^{-10} \mathrm{~mol} /\left(\mathrm{m}^{3 *} \mathrm{~s}\right)$ (calculated from [29]).

For the computation of oxygen gradients, we corrected for the difference in oxygen consumption in different layers [30]. Therefore, the explants were divided into ten different zones with the top two layers having a different consumption rate compared to the bottom eight layers. The rationale behind this division was described by Heywood et al, where oxygen consumption rates were measured in superficial and deep zone chondrocytes. Superficial zone cells were isolated from the top $20 \%$ of the tissue depth and deep zone cells from the remaining 80\% [30]. Oxygen diffusion constant $(\theta)$ in water was $3.05 \times 10^{-9} \mathrm{~m}^{2} / \mathrm{s}$ and in cartilage was $2.2 \times 10^{-9} \mathrm{~m}^{2} / \mathrm{s}[31]$. Initial oxygen concentration in the top compartment was $0.254 \mathrm{mmol} / \mathrm{L}$, representing $100 \%$ air saturation, and $0.127 \mathrm{mmol} / \mathrm{L}$ in the bottom compartment, representing $50 \%$ air saturation. The oxygen consumption rate in the top two zones was $3.53 \times 10^{-6}$ $\mathrm{mol} /\left(\mathrm{m}^{3 *} \mathrm{~s}\right)$ and in the bottom eight zones $6.758 \times 10^{-6} \mathrm{~mol} /\left(\mathrm{m}^{3 *} \mathrm{~s}\right)$. Consumption rates were calculated after Heywood et al [30].

It has to be noted that for glucose the consumption rate was assumed homogenous throughout the cartilage. However, as oxygen consumption rates are different in various layers, the same probably also holds for glucose consumption, but this is not supported by literature data. 


\section{Explants culture}

\section{Static culture}

Cartilage explants were transferred to polycarbonate 4-chamber (comparable to figure $1 \mathrm{C}$ ) inserts and placed in the static bioreactor (figure 1B). A ring was used to separate the two medium compartments and the bioreactor was placed in a custom made 4-well plate. The top and bottom compartments were filled with differentiation medium (DMEM, 100U/mL penicillin $/ 100 \mu \mathrm{g} / \mathrm{mL}$ streptomycin, $20 \mathrm{mM}$ ascorbic acid, $40 \mu \mathrm{g} / \mathrm{mL}$ proline, $100 \mu \mathrm{g} / \mathrm{mL}$ sodium pyruvate, $1 \%$ Insulin Transferrin and Selenium premix, ). In specific experiments, medium in the top compartment was supplemented with $1,5 \mathrm{mg} / \mathrm{mL}$ hyaluronidase or collagenase. Volumes used were identical to those used in the dynamic bioreactor. Explants were cultured for 7 days without medium change in a humidified atmosphere at $37^{\circ} \mathrm{C}$.

\section{Dynamic culture}

Cartilage explants in the 4-chamber polycarbonate inserts were placed between a compression module insert (figure 1D) and a perforated cover plate in the bioreactor chamber (figure 1A). The reactor was connected to a syringe pump with syringes of $10 \mathrm{~mL}$ creating two separate medium compartments. Both compartments were filled with $30 \mathrm{~mL}$ of differentiation medium saturated with $20 \%$ air. The concentration of glucose in the top compartment was $25 \mathrm{mmol} / \mathrm{L}$ and in the bottom compartment was $0 \mathrm{mmol} / \mathrm{L}$. Medium flow was $0.5 \mathrm{~mL} / \mathrm{min}$. The culture was performed for 3 days at $37^{\circ} \mathrm{C}$ in an incubation unit, which is previously described [12].

\section{Viability}


Cell viability in the chondral and osteochondral model was assessed after 24 hours of culture with 3 cycles of 1 hour compression followed by 7 hours of rest with a live/dead assay according to manufacturer's protocol (Invitrogen, New York, USA). Live (green) and dead (red) cells were visualized with a separate FITC and Texas Red filters on a fluorescence microscope (Nikon Eclipse E600, USA) and microphotographs were taken with Qcapture acquisition software. These pictures were overlaid in Photoshop and the areas of dead cells and the total area were analyzed with ImageJ.

\section{Histology}

For histology, explants were dissected top to bottom and fixed in $10 \%$ buffered formalin at $4^{\circ} \mathrm{C}$ overnight and decalcified in $12.5 \%$ ethylenediaminetetraacetic acid (EDTA) dissolved in $\mathrm{H}_{2} \mathrm{O}(\mathrm{pH}=8.0)$ at $4^{\circ} \mathrm{C}$ for 4 weeks. The EDTA solution was refreshed every 7 days. Decalcified explants were embedded in Cryomatrix ${ }^{\mathrm{TM}}$ (Thermo Fisher Scientific, USA). The cryomatrix blocks were sectioned in a cryotome into $10 \mu \mathrm{m}$ thick longitudinal sections and then mounted onto Superfrost ${ }^{\circledR}$ Plus (Thermo Fisher Scientific, USA) glass slides.

\section{Safranin-O staining}

Safranin-O staining was performed as previously described [32]. In short, sections were hydrated for 10 minutes in demi water and stained with Fast Green for 3 minutes, rinsed in $1 \%$ acetic acid and subsequently stained with Safranin-O for 5 minutes and dehydrated in $96 \% \mathrm{EtOH}, 100 \% \mathrm{EtOH}$ and xylene for 2 minutes each. Section were dried and mounted with mounting medium. 


\section{Picrosirius Red}

To visualize collagen fibers sections were stained with the Picrosirius Red staining kit (BioSciences, San Jose, USA) according to the manufacturer's protocol. Shortly, sections were hydrated for 10 minutes in demi water, stained with haematoxylin for 8 minutes and rinsed in distilled water followed by staining with picrosirius red. The stained sections were washed in $70 \% \mathrm{EtOH}$ for 45 seconds and dehydrated in a graded ethanol series. Collagen fibrils were visualized using a Nikon polarization filter.

Microphotographs were taken using a light microscope (Nikon Eclipse E600, USA) and Qcapture acquisition software. Image analysis was performed using Image $\mathrm{J}$ software

\section{Glucose analysis}

After 3 days of dynamic culture, explants were physically separated into a top, middle and bottom part, weighed and, after mincing, dissolved in $50 \mu \mathrm{L}$ of PBS. After 3 days of incubation at room temperature the glucose concentration was analyzed using a Vitros DT60II medium analyzer (Ortho-Clinical Diagnostics, USA), assuming that chondrocytes were inactive and not consuming glucose. Ten samples per zone were analyzed and glucose content was corrected for the weight of the cartilage.

\section{Statistical analysis}

Statistical analysis was performed using a one-way ANOVA using SPSS version 19 followed by Tukey post-hoc testing. Differences were considered statistically significant at $\mathrm{P}<0.05$ and are indicated with an asterisk. 


\section{Results}

\section{Computational modeling of oxygen and glucose gradients}

To predict whether our bioreactor design allowed for the formation of biological relevant gradients of bioactive molecules a computational model was made using glucose and oxygen as representative molecules. We modeled the design with the square cartilage only explants. Additionally, two different types of inlets were modeled, namely a horizontal inlet and a diagonal inlet. The model predicted that with both inlets biological relevant gradients were formed for both oxygen and glucose (Figure 2). Interestingly, the two types of inlets created gradients that differed in concentration difference and distribution. The gradients generated by the horizontal inlets were larger than those created by the diagonal inlets. Thus, by a simple adaptation in the orientation of the inlets the concentration gradients within the explant could be varied in a controlled manner.

\section{Experimental measurement of glucose gradient in explants.}

In the square cartilage explants without compression, some cell death was observed at the lateral sites of the explant likely due to sample processing (insert ref). Hardly any cell death was observed in the top, in the center or bottom of the cartilage explant either in static or dynamic culture. Cartilage explants were successfully cultured for up to 7 days without loss of viability (data not shown). This showed that limitation of diffusion by confinement of the explant was not a limiting factor for chondrocyte survival.To validate the predicted gradient (Figure 3A), cartilage explants were subsequently dynamically cultured for 3 days using medium containing 
$25 \mathrm{mmol} / \mathrm{L}$ of glucose in the top compartment and medium containing $0 \mathrm{mmol} / \mathrm{L}$ of glucose in the bottom compartment. Figure 3B shows the glucose concentration in the top, middle and bottom layer of the explants after 3 days of culture. The concentration decreased with depth and the concentration in the top layer significantly differed from the one in the bottom layer. The decrease in concentration from top to bottom suggests that a gradient can be formed during 3 days of culture. However, the experimental values did not fully comply with the computational model. This is most likely due to the fact that the diffusion coefficient of glucose through the tissue, in contrast to the model, is not homogenous, but differs from zone to zone. Another possibility could be that permeability of the tissue is higher than what is assumed in our model $[33,34]$.Regardless, we validated the formation of a biologically relevant gradient using the dual flow-approach.

\section{Gradient formation of cartilage degrading enzymes}

We next assessed whether gradients in the explants could also be obtained by larger molecules like proteins. For this we used enzymes involved in cartilage degradation as model compounds. Several enzymes involved in cartilage degradation enter the cartilage via the synovial fluid $[35,36]$. In our models we mimicked this by culturing explants using two physically separated medium compartments that are located on either side. The medium on the synovial side of the explant was supplemented with either hyaluronidase or collagenase. After 7 days of culture, explants were stained for sGAGs and collagens. Safranin-O and Picrosirius Red staining revealed a loss of sulphated GAGs or collagen, respectively, at the synovial side of the cartilage as compared to a freshly isolated sample (Figure $4 \mathrm{~A}$ and C). Moreover, there was no loss at the lateral sides or the subchondral side indicating the absence of leakage. 
Quantification of the histological section showed a significant decrease in matrix components (Figure 4B and D). In addition, no matrix degradation on the lateral sides of the explants could be observed. This strongly suggests that the system does not suffer from leakage ensuring that the additives from the top compartment can only reach the bottom compartment via diffusion through the tissue. Taken together, our bioreactor set up this system allows for the creation of gradients of molecules of choice through cartilage explants.

Compression and cell viability.

We next examined the effect of compression on chondrocyte viability. Square cartilage explants were compressed for 24 hours ( 1 hour compression, 7 hours rest with $0,25 \mathrm{MPa}$ compressive load $(\sim 2.5 \%$ strain) at $0,33 \mathrm{~Hz})$ and a live dead assay was performed. In remarkable contrast to static or dynamic culture of cartilage explants which showed negligible cell death in top and bottom cell layers of the explant (data not shown), considerable cell death was observed after 24 hours at the top and bottom of the explant (Figure 5). Most cell death was noted at the top edges of the explant (Figure 5, arrow), while cell death in the top central part of the explant was reduced (Figure 5, asterisk). Chondrocytes in the middle of the explant did not show evidence of increased cell death. Cell death at the lateral sites was more pronounced than in static or dynamic culture.

We reasoned that the square compression plate which tight fitted in the cartilage inserts used for explant culture was responsible for the increased cell death in particular at the top lateral sites of the explant. This design was not compatible with tissue expansion and deformation upon compression likely resulting in high stresses at the borders of the explant resulting in the observed cell death. To test this we 
redesigned the inset for explant culture in the bioreactor and the compression module. The new inset is designed for culturing cylindrical osteochondral plugs with a width of $7 \mathrm{~mm}$. The top of the compression module was rounded and made out of teflon instead of metal. The rounded tip is compatible with tissue deformation at the lateral sides. Like the square cartilage explants, the cylindrical osteochondral plugs could be isolated and cultured in the bioreactor either statically or dynamically without noticeable cell death of chondrocytes anywhere in the explant for up to 2 weeks (data not shown). Interestingly and in marked contrast to the previous design. 24 hours of compression ( 1 hour compression, 7 hours rest with $0,25 \mathrm{MPa}$ compressive load $(\sim 2.5 \%$ strain $)$ at $0,33 \mathrm{~Hz})$ dramatically reduced the thickness of layers with cell death at the top, bottom, and lateral sites of the cartilage explants from on average $8 \%$ to less than $0.2 \%$ of total construct height or width (Figure 5 ). Also the cell death at the top lateral sites was markedly reduced. 


\section{Discussion}

This study reports on the development of a novel bioreactor for cartilage tissue engineering. Our design is unique due to the incorporation of both confined cyclic mechanical loading and nutrient supply via diffusion from both the top and bottom separately. This form of nutrient supply resembles the natural situation in native cartilage. The combination of both features in one bioreactor set up generates a system that more closely resembles the native environment of articular cartilage compared to previously reported bioreactors [22-24]. We successfully succeeded in culturing cartilage explants and osteochondral plugs in our bioreactor for up to 2 weeks in static or dynamic flow without any significant cell death in the cartilage layers. In the square cartilage explants some cell death was noted in peripheral layers at the lateral sites of the construct. These likely arise as a consequence of cutting the cartilage $[37,38]$. The data do indicate, however, that in our design diffusion of nutrients, oxygen and waste products from top or bottom is sufficient to keep middle zone chondrocytes alive.

Computational modeling predicted that our design is suitable for the formation of gradients of small molecules like glucose and oxygen in cartilage explants. The formation of these these gradients can be manipulated with simple modifications in the design by changing the position of the inlets for dynamic flow or by varying the nutrient concentrations above or below the construct. The design allows to explore the effect of gradients of a wide variety of molecules on healthy and tissue engineered cartilage $[31,39]$ and to test the hypothesis that gradients of signaling molecules are important for reconstructing cartilage's zonal architecture. For nutrients, like glucose, there is little known how the gradient in articular cartilage looks like. Since middle and deep zone chondrocytes rely on diffusion from either the 
synovial fluid or subchondral bone compartment it is likely that such gradient exist. In line with this, Heywood et al described differences in metabolic responses in chondrocyte subpopulations, which may be involved in establishing specific zonal phenotypes [31, 40]. We performed two experiments to demonstrate the formation of a gradient in tissue explants cultured in our bioreactor by varying the concentration of compounds in the top and bottom compartment. First we used glucose as a model compound for nutrients and added a concentration of $25 \mathrm{mM}$ in the top compartment and $\mathrm{OmM}$ in the bottom compartment. Experimentally a glucose gradient was detected across the cartilage explant. This experimental gradient did it did not exactly match the predicted one by computational modeling. In computational models it is assumed that the glucose consumption rate in cartilage is homogenous, however in reality it is not [30]. This is an important notion as it may also hold true for other nutrients as well as growth factors. For secreted soluble growth factors the abundance, their zonal distribution and the affinity and abundance of receptors for these factors will also play an important role. All these parameters, of which many are still unknown will affect the diffusion of growth factors and nutrients and thus the nature of a gradient. Fundamental knowledge regarding diffusion coefficients of many nutrients and growth factors in cartilage is also lacking. All these factors likely contribute to the the deviation in our modeled glucose gradient versus the experimentally determined values. It should be noted that the computational modeling and the experimental gradient across a cartilage explant were studied in the absence of mechanical load. Mechanical compression likely affects the diffusion of molecules in the cartilage as it results in water displacement. However, the true nature of the effects remains largely unknown. Our bioreactor can function as an enabling technology that can facilitate in depth studies of these topics. 
Besides glucose, two proteins were used to study the formation of a gradient, namely hyaluronidase and collagenase. These enzymes were added in the top compartment mimicking their presence in synovial fluid as in osteoarthritis [35, 36]. By studying the depletion of sGAGs and collagen from the tissue using histological staining the formation of gradients could be easily studied. The results show that the absence of leakage at the lateral sites of the explant and the formation of a top down gradient. They demonstrate the feasibility of our design to build gradients of proteins in explants.

Next to culturing explants in an environment that mimics the natural situation in several key features, we hypothesize that creating gradients in a controlled way can contribute to the development of tissue engineered grafts closely resembling cartilage's natural architecture in vitro. Current research focuses on elucidating what concentration ranges stimulate the expression of zone specific proteins like superficial zone protein, cartilage intermediate layer protein and cartilage oligomeric matrix protein [41]. Introduction of gradients may contribute to native matrix distribution in tissue engineered cartilage. In addition, the effect of compression on these developing constructs can be studied in depth using this bioreactor. Although rotating compression can best simulate the natural load of the joint, it is less controllable than confined or sliding compression, as different forces are exerted on the tissue (indentation and rotation) and each can have different effects on the tissue. Since load is an important feature in cartilage development, we complemented the two compartment bioreactor with confined compression. When confined compression was applied to square cartilage explants using a tight fit perforated metal plate substantial cell death was noted at the periphery of the explant particularly at the top lateral sites. This is likely explained by the design of the bioreactor's insets which did 
not allow tissue deformation as response upon load resulting in high stresses at the periphery of the construct and consequently cell death. This issue could be easily solved by adapting the inset for explant culture as well as the module used for confined compression. The new inset is compatible with the culture of cylindrical cartilage explants. Cylindrical osteochondral plugs can be easily isolated using standardized orthopedic equipment. For confined compression a rounded module was used made out of teflon. The mechanical properties of teflon better resemble the mechanical properties of native cartilage compared to metal. In addition, the rounded surface of the tip is compatible with tissue expansion at the lateral sites upon load. Using this new design negligible cell death was observed at the periphery of the cartilage explant. Besides the possibility for tissue expansion at the lateral sites upon load, the use of Teflon instead of metal for compression and the cylindrical shape of the explant may also contribute to improved cell survival upon load. Using this new design it will be possible to culture chondral or even osteochondral explants or tissue engineered constructs for prolonged periods of time in the bioreactor and study the role of compression.

Our system is advantageous over current mechano-perfusion bioreactor systems for cartilage tissue engineering as it is not based on convection but on diffusion from physiologically relevant sides. This more closely mimics the natural environment of articular cartilage. This strategy allows us to take advantage of a two compartment system generating biologically relevant gradients in a tissue engineered construct or explant culture. These gradients can be modulated by applying different concentrations of molecules of interest in each compartment. In addition, the gradient can be controlled by changing the orientation of the medium inlets. 
Using simple modifications in our system we can include other tissues such as bone and synovial tissue. This would further increase the resemblance of the microenvironment generated in our system to the native environment of cartilage. Moreover, with the addition of bone by explant culture of osteochondral plugs and/or synovium it is able to function as a more accurate ex-vivo model for degenerative cartilage diseases such as osteoarthritis when compared to standard explants cultures. Particularly, it incorporates the idea that osteoarthritis is not a single tissue disease but a joint disease [42-45].

In summary, we have developed a unique bioreactor system that captures two key features of cartilage's natural environment. It has the ability to create gradients of nutrients and growth factors and apply confined cyclic compression. We believe that this will aid in development of an in vitro environment for cartilage homeostasis facilitating neo-cartilage formation. Therefore, our bioreactor holds the potential to grant deeper insight in fundamental concepts of cartilage biology, model degenerative cartilage diseases like osteoarthritis and stimulate the development of novel therapeutics for such diseases. 


\section{Acknowledgements}

This research forms part of the Project P2.02 OAcontrol of the research program of the BioMedical Materials institute, co-funded by the Dutch Ministry of Economic Affairs, Agriculture and Innovation. The authors gratefully acknowledge the support of the TeRM Smart Mix Program of the Netherlands Ministry of Economic Affairs and the Netherlands Ministry of Education, Culture and Science.

No competing financial interests exist. 


\section{References}

1. Linden, B., Osteochondritis dissecans of the femoral condyles: a long-term follow-up study. J Bone Joint Surg Am, 1977. 59(6): p. 769-76.

2. Messner, K. and J. Gillquist, Cartilage repair. A critical review. Acta Orthop Scand, 1996. 67(5): p. 523-9.

3. Kon, E., et al., Articular Cartilage Treatment in High-Level Male Soccer Players: A Prospective Comparative Study of Arthroscopic Second-Generation Autologous Chondrocyte Implantation Versus Microfracture. Am J Sports Med, 2011.

4. Steadman, J.R., W.G. Rodkey, and K.K. Briggs, Microfracture to treat full-thickness chondral defects: surgical technique, rehabilitation, and outcomes. J Knee Surg, 2002. 15(3): p. 170-6.

5. Bartlett, W., et al., Autologous chondrocyte implantation versus matrix-induced autologous chondrocyte implantation for osteochondral defects of the knee: a prospective, randomised study. J Bone Joint Surg Br, 2005. 87(5): p. 640-5.

6. Knutsen, G., et al., Autologous chondrocyte implantation compared with microfracture in the knee. A randomized trial. J Bone Joint Surg Am, 2004. 86-A(3): p. 455-64.

7. Knutsen, G., et al., A randomized trial comparing autologous chondrocyte implantation with microfracture. Findings at five years. J Bone Joint Surg Am, 2007. 89(10): p. 2105-12.

8. Kreuz, P.C., et al., Is microfracture of chondral defects in the knee associated with different results in patients aged 40 years or younger? Arthroscopy, 2006. 22(11): p. 1180-6.

9. Kreuz, P.C., et al., Results after microfracture of full-thickness chondral defects in different compartments in the knee. Osteoarthritis Cartilage, 2006. 14(11): p. 1119-25.

10. Mithoefer, K., et al., The microfracture technique for the treatment of articular cartilage lesions in the knee. A prospective cohort study. J Bone Joint Surg Am, 2005. 87(9): p. 1911-20.

11. Mithoefer, K., et al., High-impact athletics after knee articular cartilage repair: a prospective evaluation of the microfracture technique. Am J Sports Med, 2006. 34(9): p. 1413-8.

12. Janssen, F.W., et al., A perfusion bioreactor system capable of producing clinically relevant volumes of tissue-engineered bone: in vivo bone formation showing proof of concept. Biomaterials, 2006. 27(3): p. 315-23.

13. Santoro, R., et al., Bioreactor based engineering of large-scale human cartilage grafts for joint resurfacing. Biomaterials, 2010. 31(34): p. 8946-52.

14. Martin, I., D. Wendt, and M. Heberer, The role of bioreactors in tissue engineering. Trends Biotechnol, 2004. 22(2): p. 80-6.

15. Huang, C.Y., et al., Experimental verification of the roles of intrinsic matrix viscoelasticity and tension-compression nonlinearity in the biphasic response of cartilage. J Biomech Eng, 2003. 125(1): p. 84-93.

16. Schumacher, B.L., et al., A novel proteoglycan synthesized and secreted by chondrocytes of the superficial zone of articular cartilage. Arch Biochem Biophys, 1994. 311(1): p. 144-52.

17. Shirazi, R., A. Shirazi-Adl, and M. Hurtig, Role of cartilage collagen fibrils networks in knee joint biomechanics under compression. J Biomech, 2008. 41(16): p. 3340-8.

18. Klein, T.J., et al., Tissue engineering of articular cartilage with biomimetic zones. Tissue Eng Part B Rev, 2009. 15(2): p. 143-57.

19. Imhof, H., et al., Subchondral bone and cartilage disease: a rediscovered functional unit. Invest Radiol, 2000. 35(10): p. 581-8.

20. Pan, J., et al., In situ measurement of transport between subchondral bone and articular cartilage. J Orthop Res, 2009. 27(10): p. 1347-52.

21. Chang, C.H., et al., Cartilage tissue engineering on the surface of a novel gelatin-calciumphosphate biphasic scaffold in a double-chamber bioreactor. J Biomed Mater Res B Appl Biomater, 2004. 71(2): p. 313-21.

22. Demarteau, O., et al., Development and validation of a bioreactor for physical stimulation of engineered cartilage. Biorheology, 2003. 40(1-3): p. 331-6. 
23. Kock, L.M., et al., Tuning the differentiation of periosteum-derived cartilage using biochemical and mechanical stimulations. Osteoarthritis Cartilage, 2010. 18(11): p. 1528-35.

24. Wimmer, M.A., et al., Tribology approach to the engineering and study of articular cartilage. Tissue Eng, 2004. 10(9-10): p. 1436-45.

25. Grad, S., et al., Effects of simple and complex motion patterns on gene expression of chondrocytes seeded in 3D scaffolds. Tissue Eng, 2006. 12(11): p. 3171-9.

26. Grad, S., et al., Chondrocyte gene expression under applied surface motion. Biorheology, 2006. 43(3-4): p. 259-69.

27. Allhands, R.V., P.A. Torzilli, and F.A. Kallfelz, Measurement of diffusion of uncharged molecules in articular cartilage. Cornell Vet, 1984. 74(2): p. 111-23.

28. Maroudas, A., Distribution and diffusion of solutes in articular cartilage. Biophys J, 1970. 10(5): p. 365-79.

29. Zhou, S., Z. Cui, and J.P. Urban, Nutrient gradients in engineered cartilage: metabolic kinetics measurement and mass transfer modeling. Biotechnol Bioeng, 2008. 101(2): p. 408-21.

30. Heywood, H.K., M.M. Knight, and D.A. Lee, Both superficial and deep zone articular chondrocyte subpopulations exhibit the Crabtree effect but have different basal oxygen consumption rates. J Cell Physiol, 2010. 223(3): p. 630-9.

31. Malda, J., et al., Oxygen gradients in tissue-engineered PEGT/PBT cartilaginous constructs: measurement and modeling. Biotechnol Bioeng, 2004. 86(1): p. 9-18.

32. Moreira Teixeira, L.S., et al., High throughput generated micro-aggregates of chondrocytes stimulate cartilage formation in vitro and in vivo. Eur Cell Mater, 2012. 23: p. 387-99.

33. Korhonen, R.K., et al., Fibril reinforced poroelastic model predicts specifically mechanical behavior of normal, proteoglycan depleted and collagen degraded articular cartilage. J Biomech, 2003. 36(9): p. 1373-9.

34. Seifzadeh, A., D.C. Oguamanam, and M. Papini, Evaluation of the constitutive properties of native, tissue engineered, and degenerated articular cartilage. Clin Biomech (Bristol, Avon), 2012. 27(8): p. 852-8.

35. Fernandes, J.C., J. Martel-Pelletier, and J.P. Pelletier, The role of cytokines in osteoarthritis pathophysiology. Biorheology, 2002. 39(1-2): p. 237-46.

36. Kapoor, M., et al., Role of proinflammatory cytokines in the pathophysiology of osteoarthritis. Nat Rev Rheumatol, 2011. 7(1): p. 33-42.

37. Amin, A.K., et al., Chondrocyte death in mechanically injured articular cartilage--the influence of extracellular calcium. J Orthop Res, 2009. 27(6): p. 778-84.

38. Secretan, C., K.M. Bagnall, and N.M. Jomha, Effects of introducing cultured human chondrocytes into a human articular cartilage explant model. Cell Tissue Res, 2010. 339(2): p. 421-7.

39. Zhou, S., Z. Cui, and J.P. Urban, Factors influencing the oxygen concentration gradient from the synovial surface of articular cartilage to the cartilage-bone interface: a modeling study. Arthritis Rheum, 2004. 50(12): p. 3915-24.

40. Heywood, H.K., D.L. Bader, and D.A. Lee, Glucose concentration and medium volume influence cell viability and glycosaminoglycan synthesis in chondrocyte-seeded alginate constructs. Tissue Eng, 2006. 12(12): p. 3487-96.

41. Coates, E.E. and J.P. Fisher, Phenotypic variations in chondrocyte subpopulations and their response to in vitro culture and external stimuli. Ann Biomed Eng, 2010. 38(11): p. 3371-88.

42. Castaneda, S., et al., Subchondral bone as a key target for osteoarthritis treatment. Biochem Pharmacol, 2011.

43. Findlay, D.M., Vascular pathology and osteoarthritis. Rheumatology (Oxford), 2007. 46(12): p. $1763-8$.

44. Shim, V., et al., A multiscale framework based on the Physiome markup languages for exploring the initiation of osteoarthritis at the bone cartilage interface. IEEE Trans Biomed Eng, 2011. 
Page 25 of 35

45. Aspden, R.M., Osteoarthritis: a problem of growth not decay? Rheumatology (Oxford), 2008. 47(10): p. 1452-60.

\section{Figure legends}




\section{Bioreactor for dynamic culture}

Bioreactor for static culture
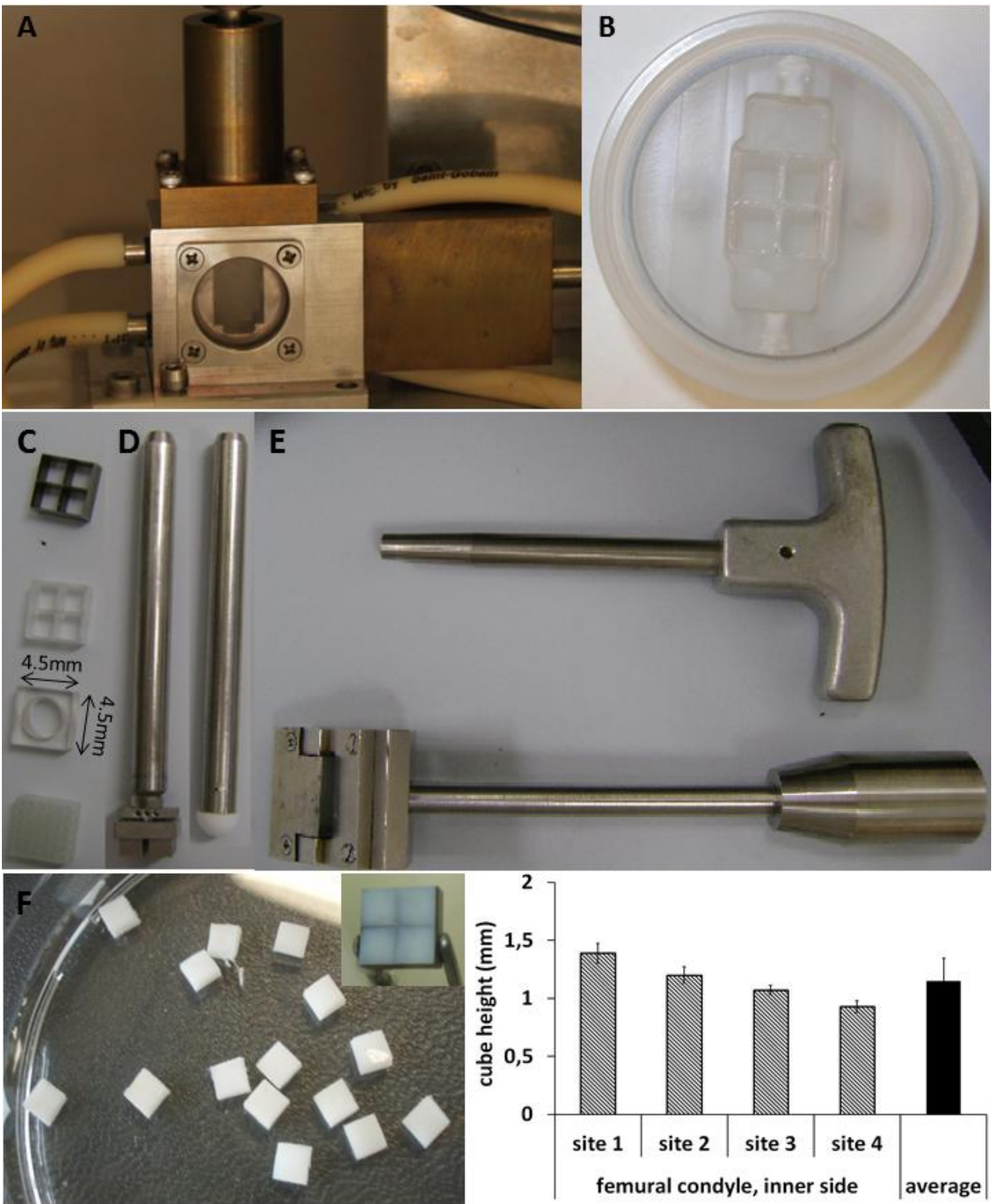

Figure 1

Figure 1: A novel bioreactor for articular cartilage explants. (A) A lateral view of the mechano-dual flow bioreactor. (B) A top view of dual compartment bioreactor for 
static culture. (C) A knife (top) for explant punch harvesting, an insert to accommodate squared cartilage explants (second from top) for bioreactor culture, an insert to house a cylindrical osteochondral explant (second from bottom) and a perforated plate to support explants (bottom). (D) A 4-module compression insert for the square explant samples (left) and a rounded compression module for the cylindrical explants (right). (E) A plunger to harvest osteochondral explants (top) and a cartilage slicer to slice off full thickness cartilage slices (bottom). (G) Reproducible standardized articular cartilage cubes are obtained using the cartilage slicer in combination with the square knife (left). The inset shows explants in the knife, The height of the cubes depended on the region of harvest. Cubes of one region were equal in height (right). 

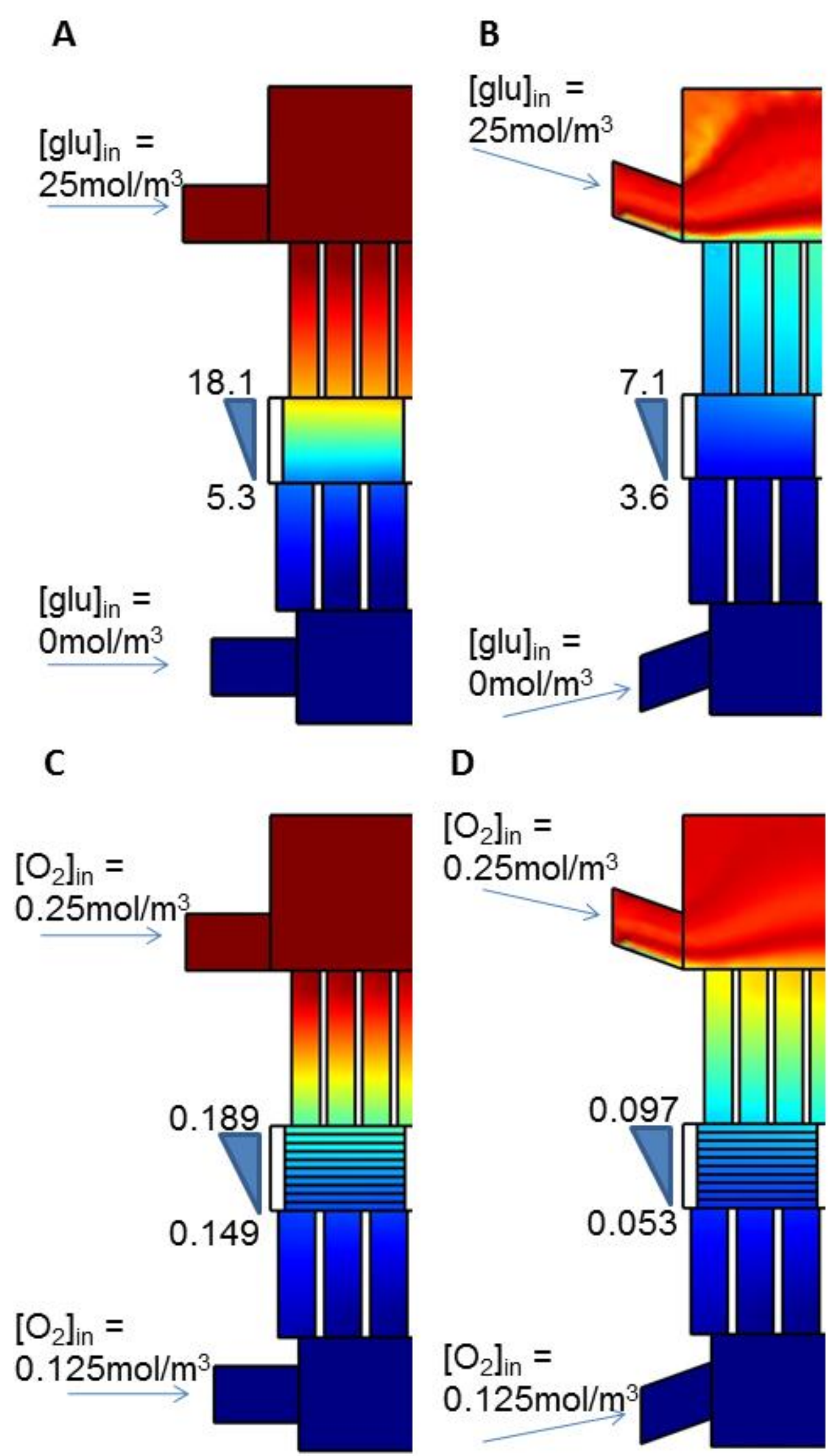
Figure 2: Computational modeling of the glucose and oxygen gradient formation within the explants in the dual flow bioreactor with two different designs. Prediction of the glucose gradient when horizontal $(A)$ or diagonal $(B)$ inlets are used. Prediction of the oxygen gradient when horizontal (C) or diagonal (D) inlets are used. Dark red represents a [glu] of $25 \mathrm{mmol} / \mathrm{L}$ and dark blue represents a [glu] of $0 \mathrm{mmol} / \mathrm{L}$ and concentration decreases from dark red via orange and yellow to dark blue. The same holds for the $\left[\mathrm{O}_{2}\right]$. 


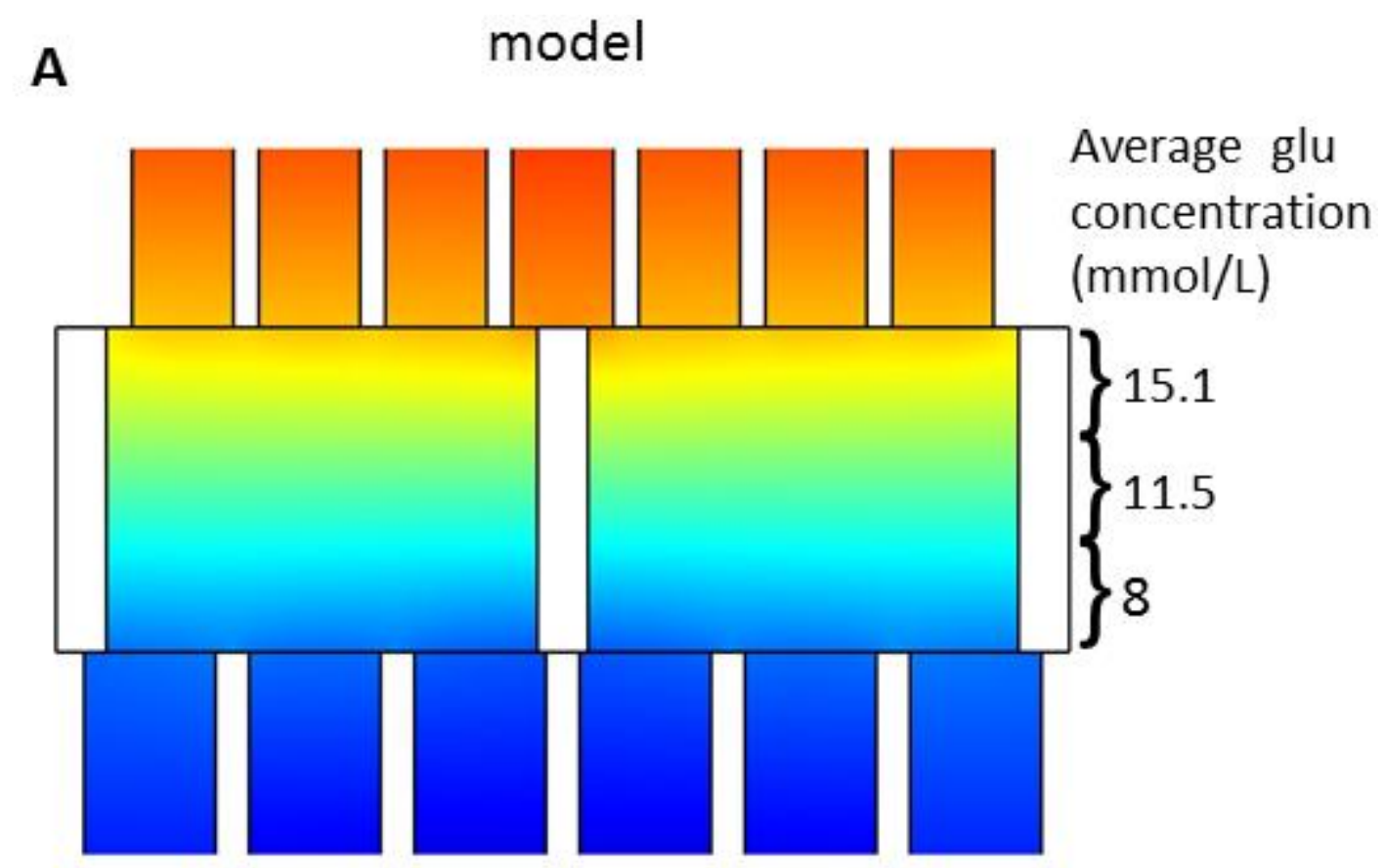

experiment

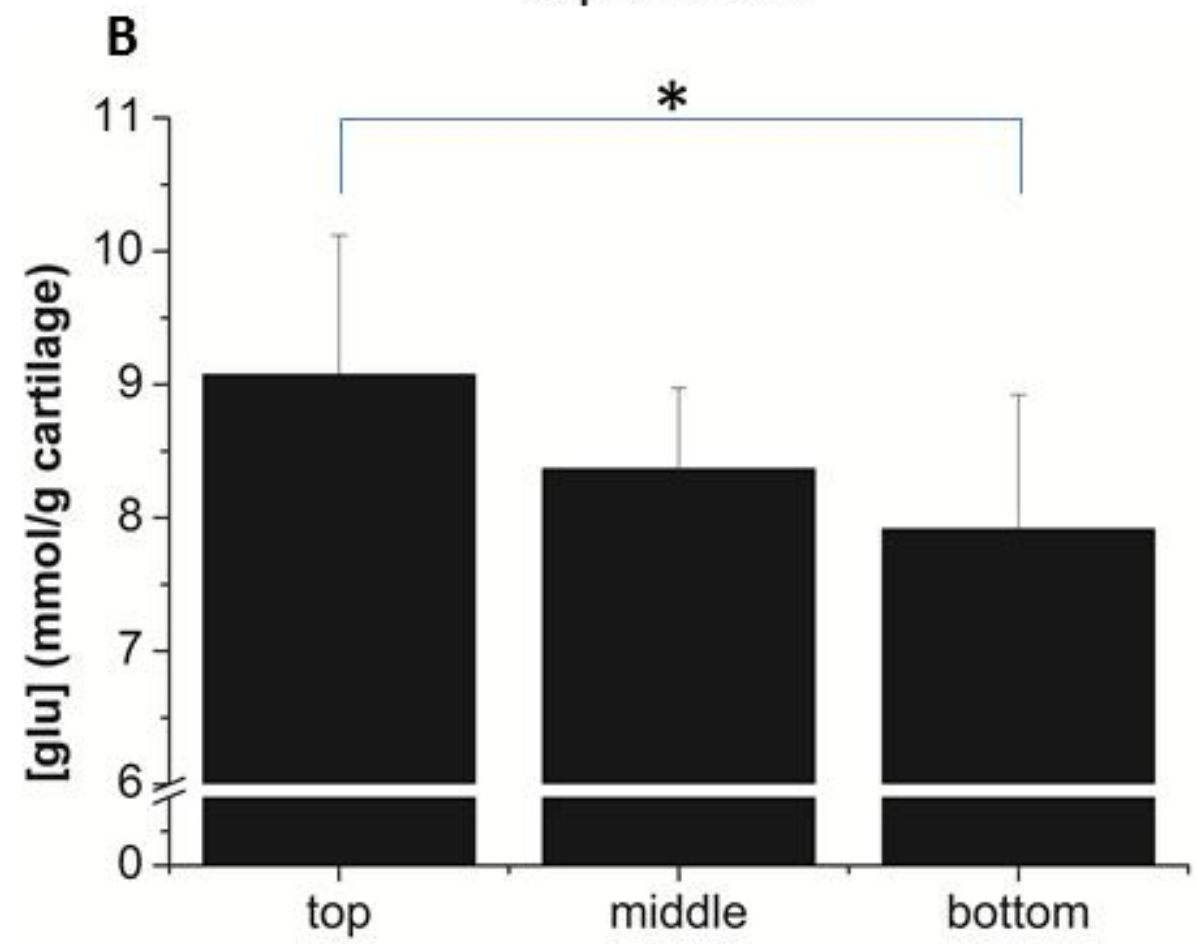

Figure 3: Predicted and experimental glucose concentrations in different zones of articular cartilage. (A) Quantification of the modeled gradients, (B) Quantification of the glucose concentration in different zones after 3 days of dynamic culture 
$\left({ }^{*}=P<0,05, n=10\right)$. The data is represented as the mean glucose concentration/gram cartilage $+/$ - the standard deviation of the mean. 


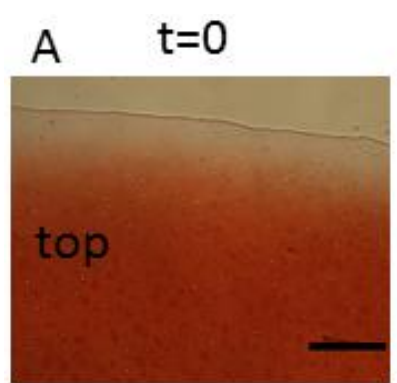

\section{hyaluronidase}
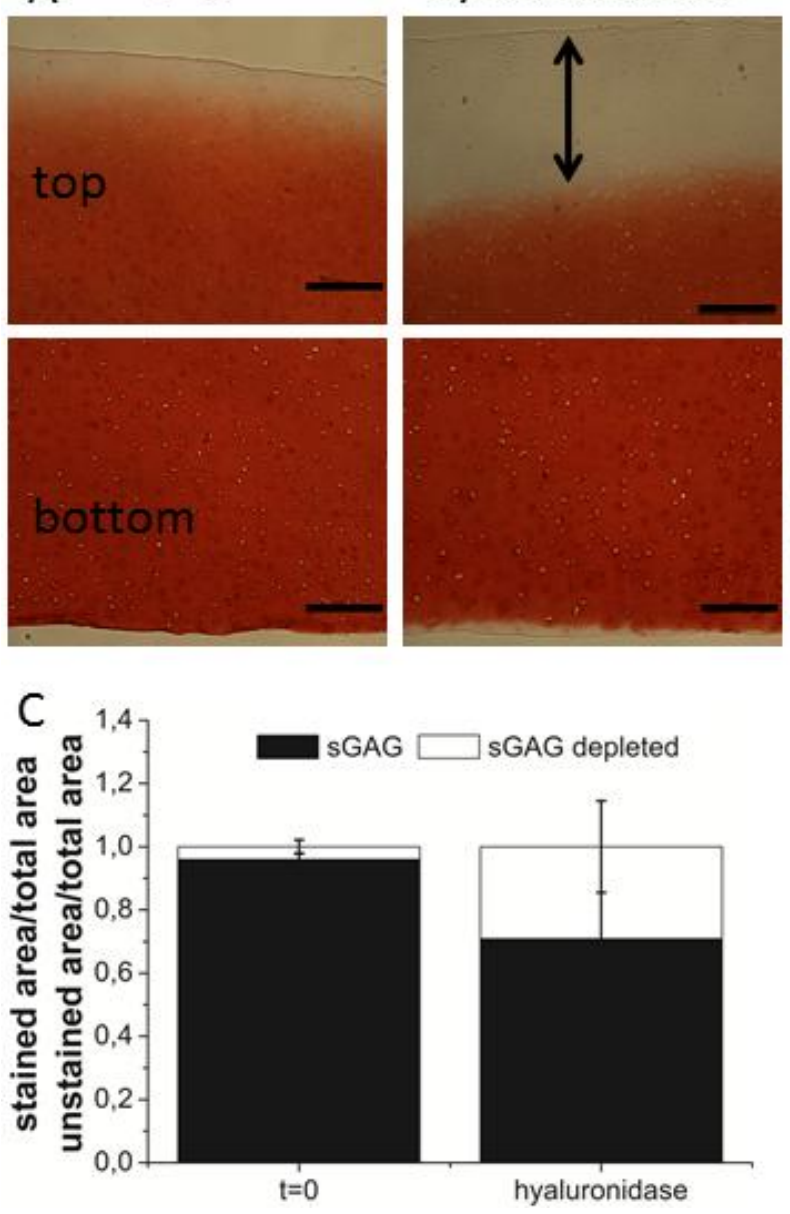
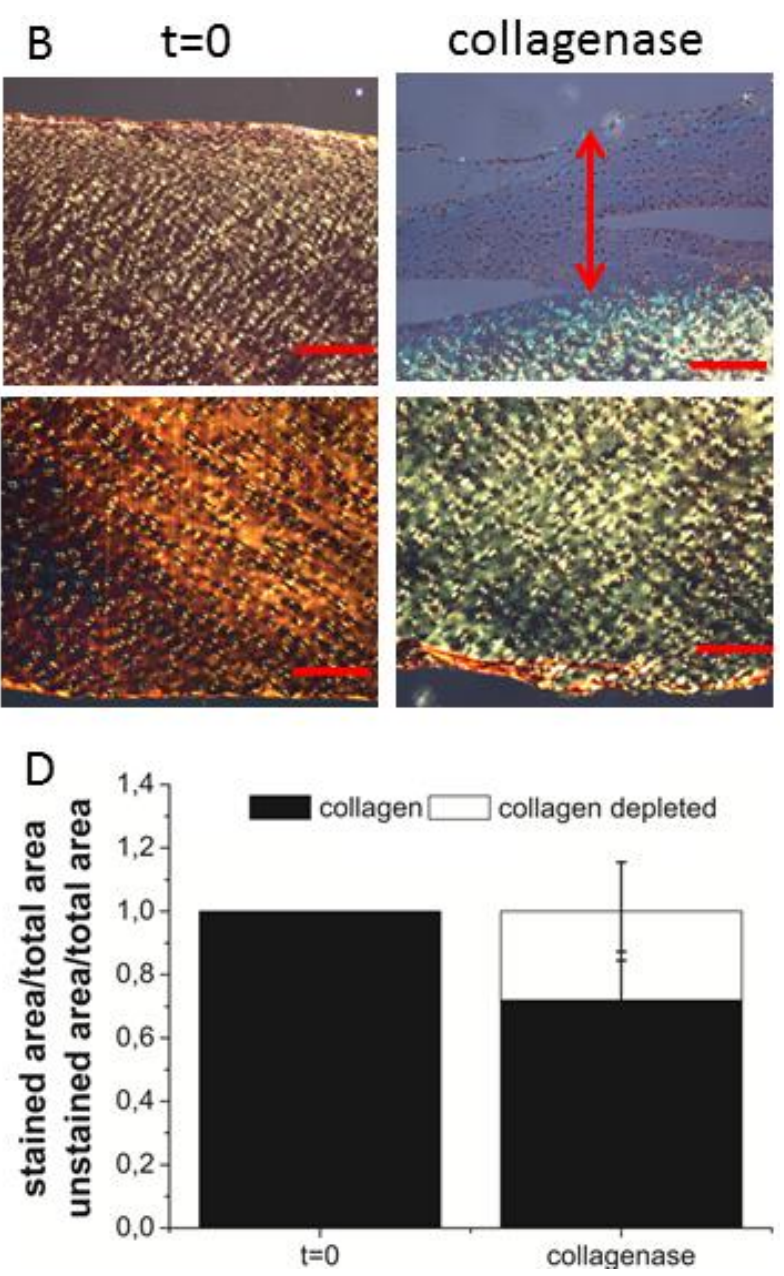

Figure 4: Matrix degradation in a static dual compartment system. (A) Safranin-O stained explants cultured without and with hyaluronidase on top. A representative example out of 9 slides is shown. (B) Picrosirius Red stained explants cultured without and with collagenase on top (polarized light pictures). A representative example out of 7 slides is shown. (C) Quantification of GAG depletion in histological samples ( $n=9$ slides from 4 explants). (D) Quantification of collagen depletion in histological samples ( $n=7$ slides from 4 explants). The upward standard error bars belong to the bars representing the nonstained parts (red) and the downward errors bars to the stained parts (blue). Black and red arrow indicate the depth of matrix degradation. 
Page 33 of 35 


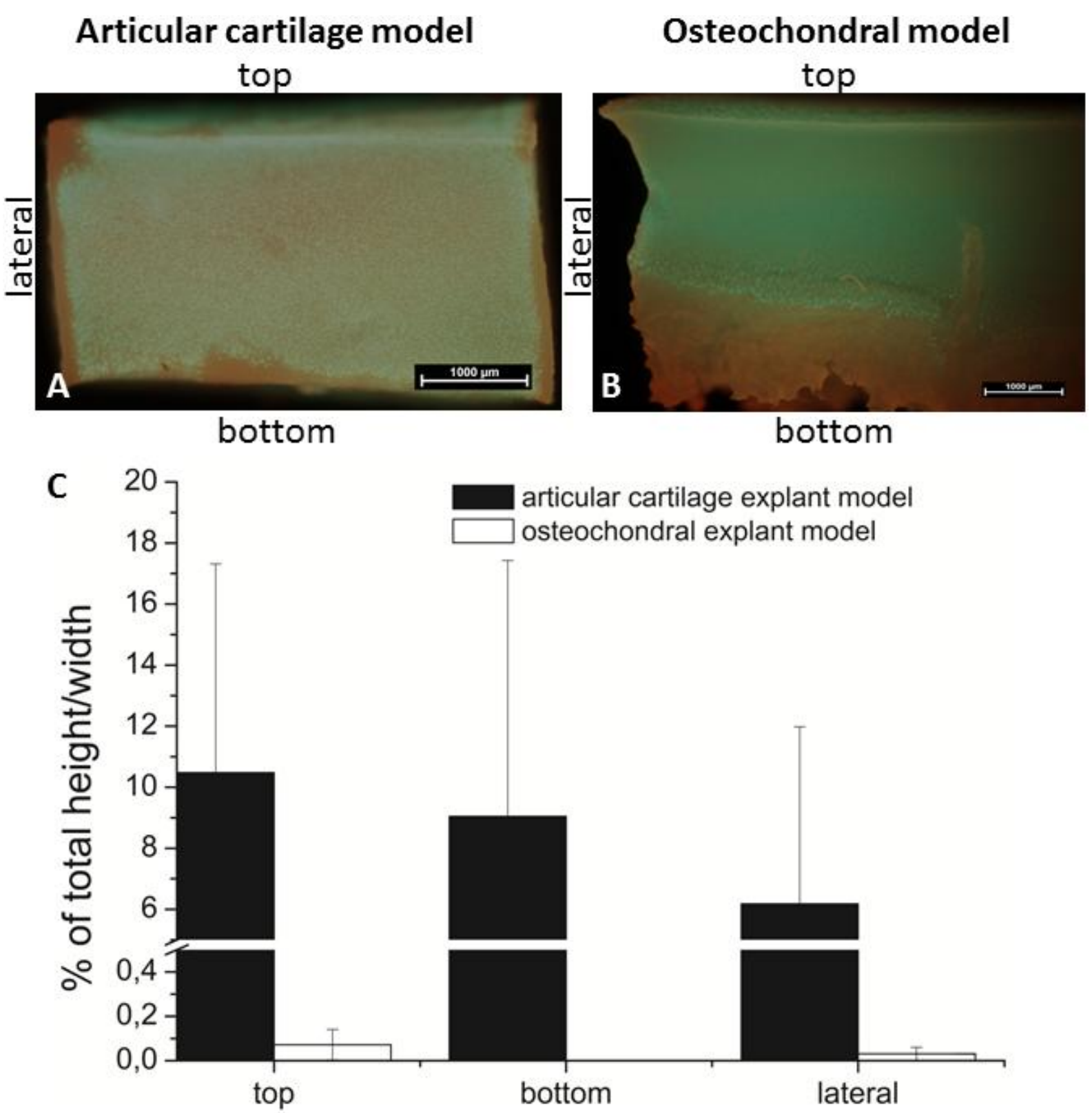

Figure 5: Design validation. Live/dead assays were performed on cartilage explants and on the cartilage in osteochondral explants after compression (24 hours (1 hour compression, 7 hours rest) with $0,25 \mathrm{MPa}$ compressive load ( 2.5\% strain) at $0,33 \mathrm{~Hz}$ ). In both constructs, cell death was only noted in layers at the periphery of the cartilage explants but not in the center. A) Live/dead staining of a compressed cartilage explant. The arrow indicates the area where most cell death is observed. 
The asterisk indicates the top center of the explant. B) Live/dead staining of a compressed osteochondral explant. The red staining in the lower part of the explant is due to non-specific binding of the ethidium homodimer to mineralized bone matrix. A representative example out of 3 specimens is shown. C) Quantification of A and B. The thickness of the layers was measured and expressed as $\%+/$ - standard deviation of the total height at the top or bottom or width of the cartilage explant at the lateral sites. ( $n=5$ (articular cartilage model), $n=3$ (osteochondral model)). 